\title{
Editorial: Hormone Receptors and Breast Cancer
}

\author{
Antonio Brunetti ${ }^{1 *}$ and Guidalberto Manfioletti ${ }^{2 *}$ \\ ${ }^{1}$ Department of Health Sciences, University "Magna Graecia" of Catanzaro, Catanzaro, Italy, ${ }^{2}$ Department of Life Sciences, \\ University of Trieste, Trieste, Italy
}

Keywords: estrogen receptors, steroid receptors, tyrosine kinase receptors, hormone resistance, triple negative breast cancer

\section{Editorial on the Research Topic}

\section{Hormone Receptors and Breast Cancer}

Breast cancer (BC) is the most commonly diagnosed cancer among women worldwide and one of the leading cause of cancer-related deaths (1). The majority of BCs arise from epithelial cells, either in the ducts or lobules, as the result of genetic and epigenetic alterations, which lead to aberrant growth control and disruption of intracellular signaling. Because of this, BC is considered a heterogeneous disease with multiple sub-types, with cells of distinct origin and function $(2,3)$.

Overexpression of estrogen receptors (ERs), during BC development, has a significant impact, since $\sim 75 \%$ of BCs expresses ERs (4). ERs are transcription factors that, upon binding to the estrogen steroid hormone, migrate to the nucleus, driving specific gene expression programs that intercept growth control pathways. Therefore, inhibiting ER, particularly ERalpha, through the use of hormone-based therapeutics such as tamoxifen, fulvestrant and aromatase inhibitors, is a primary goal of endocrine therapy in BC (5). The widespread use of these drugs as adjuvant therapies for $\mathrm{BC}$ has led to a significant reduction in mortality, although intrinsic and acquired resistance to these inhibitors may limit their efficacy. In this regard, ER mutations or loss of expression, alteration in ER coactivators/corepressors, overexpression and/or amplification of growth factor receptors which impinge on the PI3K/AKT/mTOR and RAF/MEK/ERK pathways, and alterations of cell-cycle checkpoints confer resistance to endocrine therapy (6). In recent years, our understanding of the mechanisms underlying hormone-therapy resistance in BC has improved, and this has led to the approval of three anticancer agents: the mTOR inhibitor everolimus, and the two CDK 4/6 inhibitors, palbociclib, and ribociclib (7). However, despite these successful advances, novel targets need to be identified, together with reliable and reproducible biomarkers of BC.

Apart from the intrinsic or acquired hormone-therapy resistance, there is a consistent number of breast tumors that do not express ERs. BCs lacking ERalpha and progesterone receptors, along with no overexpression of epidermal growth factor receptor 2, constitute the so-called triple-negative BC (TNBC) (8), an aggressive BC subtype that comprises $10-30 \%$ of all BCs and is associated with younger age and poorer prognosis (9). TNBCs are a group of heterogeneous BCs and represent a challenge for therapy. On the other hand, other steroid receptors, such as vitamin D receptor (10), glucocorticoid and androgen receptors $(11,12)$, as well as tyrosine kinase receptors, including IGF1 and insulin receptors (13-15), can be also expressed in breast cells, and their roles and significance in cancer cell growth might deserve further investigation.

The aim of this Research Topic was to gather up-to-date data and novel point of view in the field of hormone receptors and BC.

In addition to the classical actions of $E_{2}$-ERalpha on gene transcription, $E_{2}$-ERalpha exerts extranuclear actions that are important for activating signal transduction pathways. Among these actions, activation of the anticipatory Unfolded Protein Response (UPR) is the most recently 
described. Livezey et al. reviewed the role of mitogenic hormones in activating the UPR, an endoplasmic reticulum stress response pathway, in anticipation of a future need for increased protein folding capacity upon cell proliferation. Overexpression of UPR components strongly correlated with reduced time to recurrence, subsequent resistance to tamoxifen, and reduced survival in ERalpha-positive BC. Because of this, UPR components are considered promising targets in ERalpha-positive cancers and, in this regard, several drugs are currently being studied. Among these drug molecules, the Authors focused on a promising preclinical drug candidate, BHPI, that selectively targets ERalpha-positive cancer cells, blocking proliferation and killing tumor cells. Hence, Authors suggest exploiting the UPR pathway as a target for the development of novel anticancer drugs.

Sanchez et al. contributed a research article in which they experimentally demonstrated that gonadotrophins $\mathrm{FSH} / \mathrm{LH}$ can alter the expression of genes involved in cell adhesion, motility, and invasion via activation of their own receptors in human BC cells. This activity is mediated by moesin and FAK kinases, two actin cytoskeleton regulator proteins, whose expression and activation are modulated by FSH/LH. In a rat model of BC progression, Authors provide evidence for a direct correlation between gonadotropin levels and cancer growth. Based on this, they suggest that gonadotropins may thus influence BC progression, particularly in post-menopausal women with higher FSH/LH levels.

Bond et al. provided an overview of the functional role of ZNF423 in human malignancies, with a particular focus on its implication in hormone-responsive BC. As a zinc-finger protein, ZNF423 interacts with different activators, inhibitors, and co-interacting transcription factors, playing a key role in development and disease, including cancer. SNPs in the ZNF423 gene, which were associated with decreased risk of BC during selective ER modulators therapy, have been identified by GWAS, providing an opportunity for the identification and stratification of patients that are more likely to respond to this treatment. A role for ZNF423 in the control of the estrogen response was supported by findings indicating that ZNF423 expression can be induced by estrogens through specific response elements within the ZNF423 gene, which in turn promotes BRCA1 expression, thus modulating the DNA

\section{REFERENCES}

1. Bray F, Ferlay J, Soerjomataram I, Siegel RL, Torre LA, Jemal A. Global cancer statistics 2018: GLOBOCAN estimates of incidence and mortality worldwide for 36 cancers in 185 countries. CA Cancer J Clin. (2018) 68:394-424. doi: $10.3322 /$ caac. 21492

2. Skibinski A, Kuperwasser C. The origin of breast tumor heterogeneity. Oncogene. (2015) 34:5309-16. doi: 10.1038/onc.2014.475

3. Zardavas D, Irrthum A, Swanton C, Piccart M. Clinical management of breast cancer heterogeneity. Nat Rev Clin Oncol. (2015) 12:381-94. doi: 10.1038/nrclinonc.2015.73

4. Groner AC, Brown M. Role of steroid receptor and coregulator mutations in hormone-dependent cancers. J Clin Invest. (2017) 127:1126-35. doi: $10.1172 /$ JCI88885 damage response. In line with this is the finding that ZNF423 knocked-down cells were more sensitive to the PARP inhibitors olaparib and cisplatin, consistent with a BRCA1 decrease. In the Author's opinion future studies may establish if ZNF423 is of clinical benefit.

Cusan et al. reviewed the mutational spectrum of the CDKN1B gene that encodes for the p27 $7^{\mathrm{Kip} 1}$ protein, a key regulator of cell-cycle. Although firstly characterized as a cyclin dependent kinase-inhibitor, many studies have demonstrated that $\mathrm{p} 27^{\mathrm{Kip} 1}$ can accomplish several non-canonical functions, being able to interact with many different proteins having a central role in tumor progression (16). p2 $7^{\mathrm{Kipl}}$ is mainly regulated at post-transcriptional level; however, massive parallel sequencing demonstrated that, in some types of cancer, including $\mathrm{BC}, C D K N 1 B$ is mutated at relatively high frequency (17). By reviewing all $C D K N 1 B$ mutations in cancer, the Authors highlight that mutations affecting the $C D K N 1 B$ gene are present and can represent driver genetic lesions in the ER-positive luminal $\mathrm{BC}$ subtype. They emphasize that more work is necessary to fully clarify the role of $\mathrm{p} 27^{\mathrm{Kip} 1}$ in $\mathrm{BC}$ and other hormonedriven tumors.

In summary, these articles contribute to understanding the topic and gathering information about the molecular mechanisms that are involved in BC development and progression. A better knowledge of the mechanisms involved in the pathogenesis of $\mathrm{BC}$ could enable us to discovery new biomarkers for patient stratification and identify novel therapeutic targets.

\section{AUTHOR CONTRIBUTIONS}

All authors listed have made a substantial, direct and intellectual contribution to the work, and approved it for publication.

\section{ACKNOWLEDGMENTS}

The authors wish to dedicate this Editorial to the memory of Giovanni (Gianni) Morrone who contributed to this Research Topic and recently passed away. This work was supported from Associazione Italiana per la Ricerca sul Cancro (AIRC, IG18385) and Regione Friuli Venezia Giulia (TNBCneo and RiFT) to GM.
5. Tryfonidis K, Zardavas D, Katzenellenbogen BS, Piccart M. Endocrine treatment in breast cancer: Cure, resistance and beyond. Cancer Treat Rev. (2016) 50:68-81. doi: 10.1016/j.ctrv.2016.08.008

6. Murphy CG, Dickler MN. Endocrine resistance in hormone-responsive breast cancer: mechanisms and therapeutic strategies. Endocr Relat Cancer. (2016) 23:R337-52. doi: 10.1530/ERC-16-0121

7. Castrellon AB. Novel strategies to improve the endocrine therapy of breast cancer. Oncol Rev. (2017) 11:323. doi: 10.4081/oncol.2017.323

8. Sgarra R, Pegoraro S, Ros G, Penzo C, Chiefari E, Foti D, et al. High mobility group A (HMGA) proteins: molecular instigators of breast cancer onset and progression. Biochim Biophys Acta Rev Cancer. (2018) 1869:216-29. doi: 10.1016/j.bbcan.2018.03.001

9. Foulkes WD, Smith IE, Reis-Filho JS. Triple-negative breast cancer. N Engl J Med. (2010) 363:1938-48. doi: 10.1056/NEJMra1001389 
10. Welsh J. Function of the vitamin D endocrine system in mammary gland and breast cancer. Mol Cell Endocrinol. (2017) 453:88-95. doi: 10.1016/j.mce.2017.04.026

11. West DC, Kocherginsky M, Tonsing-Carter EY, Dolcen DN, Hosfield DJ, Lastra RR, et al. Discovery of a glucocorticoid receptor (GR) activity signature using selective GR antagonism in ER-negative breast cancer. Clin Cancer Res. (2018) 24:3433-46. doi: 10.1158/1078-0432.CCR-17-2793

12. Giovannelli P, Di Donato M, Galasso G, Di Zazzo E, Bilancio A, Migliaccio A. The androgen receptor in breast cancer. Front Endocrinol. (2018) 28:492. doi: 10.3389/fendo.2018.00492

13. Paonessa F, Foti D, Costa V, Chiefari E, Brunetti G, Leone F, et al. Activator protein-2 overexpression accounts for increased insulin receptor expression in human breast cancer. Cancer Res. (2006) 66:5085-93. doi: 10.1158/0008-5472.CAN-05-3678

14. Costa V, Foti D, Paonessa F, Chiefari E, Palaia L, Brunetti G, et al. The insulin receptor: a new anticancer target for peroxisome proliferator-activated receptor-gamma (PPARgamma) and thiazolidinedione-PPARgamma agonists. Endocr Relat Cancer. (2008) 15:325-35. doi: 10.1677/ERC07-0226
15. Ekyalongo RC, Yee D. Revisiting the IGF-1R as a breast cancer target. Precis Oncol. (2017) 1:14. doi: 10.1038/s41698-017-0017-y

16. Sharma SS, Pledger WJ. The non-canonical functions of p27 Kip1 in normal and tumor biology. Cell Cycle. (2016) 15:1189-201. doi: 10.1080/15384101.2016.1157238

17. Ellis MJ, Ding L, Shen D, Luo J, Suman VJ, Wallis JW, et al. Whole-genome analysis informs breast cancer response to aromatase inhibition. Nature. (2012) 486:353-60. doi: 10.1038/nature11143

Conflict of Interest Statement: The authors declare that the research was conducted in the absence of any commercial or financial relationships that could be construed as a potential conflict of interest.

Copyright $(2019$ Brunetti and Manfioletti. This is an open-access article distributed under the terms of the Creative Commons Attribution License (CC BY). The use, distribution or reproduction in other forums is permitted, provided the original author(s) and the copyright owner(s) are credited and that the original publication in this journal is cited, in accordance with accepted academic practice. No use, distribution or reproduction is permitted which does not comply with these terms. 